Products and Services In Practice is provided to readers using text and images from the manufacturer, supplier or distributor and does not imply endorsement by $B D J$ In Practice. Normal and prudent research should be exercised before purchase or use of any product mentioned.

Please send product and services news through to David Westgarth, BDJ In Practice via: David.Westgarth@bda.org

\section{Breathe easy}

Give staff and patients added peace of mind at this difficult time by removing potential contaminants from the air throughout your surgery.

Now available from Nuview, the JADE air purification system uses state-of-theart, multi-stage air purifying technologies from Surgically Clean Air to effectively clean and purify indoor air. It creates hourly air changes, while operating quietly and unobtrusively so as to minimise disruption in the practice.

The Jade Purifier is one of the most advanced of its kind. It features a filtration system that captures everything from dust particles to pollen, volatile organic chemicals (VOCs), mould, allergens, viruses, odours and bio-aerosols.

Make sure you, your staff and your patients can breathe easy in your practice with the JADE air purification system from Nuview.

For more information call Nuview on 01453 872266, email info@nuview-ltd. com, or visit www.nuview.co.
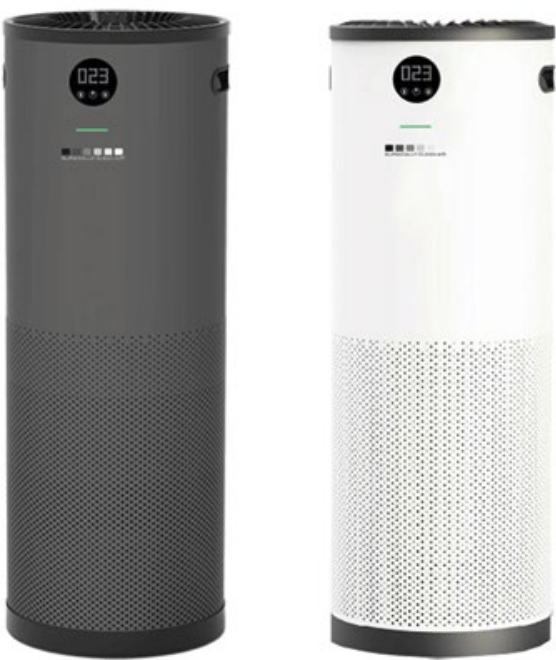

\title{
New brush launched
}

Oral-B has unveiled a new, unprecedented era in brushing with the introduction of its most advanced rechargeable electric toothbrush: Oral-B iO.

Oral-B iO represents a step forward in creating the right balance between effectiveness and experience and re-imagines how a brush performs, cleans and feels. From its revolutionary design to the harmony of its oscillation rotation and micro-vibrations technology, Oral-B iO wholly transforms brushing from a perceived chore to a pleasurable experience that patients will love.

Oral-B iO's innovative key features include:

$\rightarrow$ Deep, but uniquely gentle clean: OralB's iconic round brush head contours each tooth, while the combination of oscillation rotations with the gentle energy of micro-vibrations allows Oral-B $\mathrm{iO}$ to glide tooth by tooth for a gentle clean, even along the gum line

$\rightarrow$ Pressure optimisation: The new Smart Pressure Sensor light provides positive reinforcement and protects gums by turning green when optimal pressure is applied and red when pressure is too hard

$\rightarrow$ Precision: The new innovative Frictionless Magnetic Drive system gently transfers the energy towards the bristle tips so that it is concentrated where it is needed

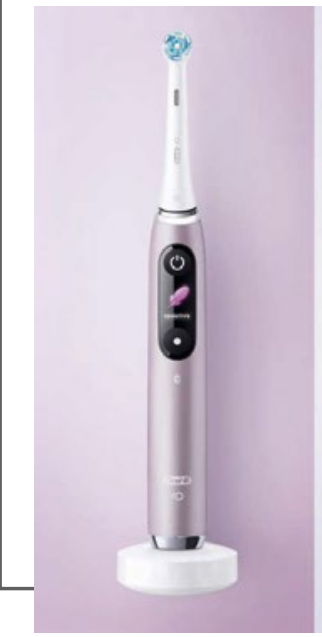

most, to experience a deep, but uniquely gentle clean. The result, Oral-B's best ever clean with six times more effective plaque removal along the gum line.

Oral-B iO's Interactive Display is equipped with easy navigation through the brush features and allows users to personalise their brushing experience through a number of settings: welcoming greetings, choice of brushing modes, battery life icons, selection of preferred colour for the icons and Smart Ring, reminders to change the brush head and language settings. It further offers an enhanced, completely holistic brushing experience with a choice of brush colours to fit the user's personality, a re-invented design and new, sleek magnetic charger, offering a full, longlasting charge in about three hours.

Oral-B iO also comes with artificial intelligence, and is equipped with position sensors located in the handle. The Series 9 includes 3D teeth tracking technology with A.I enabled brushing recognition to track the lingual and buccal areas of the three bottom and upper jaw areas, plus occlusal surfaces.

For more information about Oral-B

iO, visit https://contest.io.oralb.co.uk/ en-gb/?utm_source=OralB-Website\&utm_ medium $=$ Banner\&utm_campaign $=$ iOPrelaunch

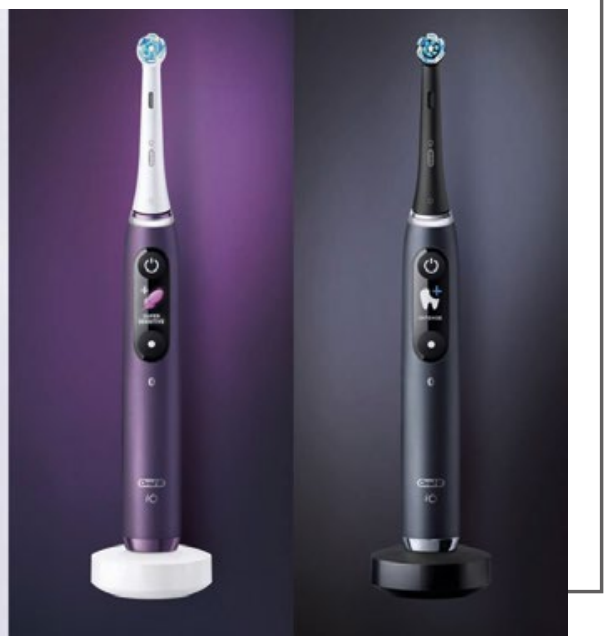

